\title{
Geomagnetic activity during the Sun-Earth connection events on April 1999 and February 2000
}

M. V. Alves, LAP - INPE, Av. dos Astronautas, 1758, PO Box 515, 12201-970, S. J. dos Campos, Brazil.

E. Echer, DGE - INPE, Av. dos Astronautas, 1758, PO Box 515, 12201-970, S. J. dos Campos, Brazil.

W. G. Gonzalez, DGE - INPE, Av. dos Astronautas, 1758, PO Box 515, 12201-970, S. J. dos Campos, Brazil.

L. A. Balmaceda, Max-Planck-Institut fuer Sonnensystemforschung, 37191 Katlenburg-Lindau, Germany

F. L. Guarnieri, Universidade do Vale do Paraiba, S. J. dos Campos, Brazil.

Copyright 2007, SBGf - Sociedade Brasileira de Geofísica

This paper was prepared for presentation at the $10^{\text {th }}$ International Congress of The Brazilian Geophysical Society held in Rio de Janeiro, Brazil, 19-22 November 2007.

Contents of this paper were reviewed by the Technical Committee of the $10^{\text {th }}$ International Congress of the Brazilian Geophysical Society and do not necessarily represent any position of the SBGf, its officers or members. Electronic reproduction, or storage of any part of this paper for commercial purposes without the written consent of the Brazilian Geophysical Society is prohibited.

\begin{abstract}
We study two geomagnetic storms: April, 16-17 1999 (peak Dst $=-91 \mathrm{nT}$ ) and February 10-12 2000 (peak Dst $=-131 \mathrm{nT}$ ). These two storms were very different in character due to the interplanetary driver: the April 1999 storm was driven by a smooth rotating $B_{z}$ field in a magnetic cloud which produced a very smooth, single-step and monotonic storm; the February 2000 storm was driven by the interaction of two shocks and two ICMES and as a consequence of the irregular and fluctuating IMF $B_{z}$ fields, this storm was quite irregular. We analyze in this work the geomagnetic activity during these two storms by using the SYM-H, $A S Y-H, K p$ and $A E$ indices.
\end{abstract}

\section{Introduction}

The Earth's magnetosphere is the region in space around our planet where the geomagnetic field dominates. It is generated by the interaction of the magnetized solar wind with the Earth's intrinsic magnetic field (Wolf, 1995; Gonzalez et al., 1999; Echer et al., 2005). The magnetosphere has been explored by several spacecraft in the last 40 years (Wolf, 1995). Solar windmagnetosphere coupling occurs predominantly through the magnetic reconnection mechanism in the diurnal magnetopause (Dungey, 1961; Gonzalez et al., 1994). Through this mechanism, the magnetosphere is driven in a disturbed state, called a magnetic or geomagnetic storm. The magnetic storm can be monitored by using ground-based magnetometers, which measure the magnetic disturbances due to enhanced current systems. Among them, the ring current can be monitored by the Dst index (Gonzalez et al., 1994).

It is the objective of the present paper to study the geomagnetic activity during two different magnetic storms: April, 16-17 1999, driven by a magnetic cloud (MC) and February 11-12 2000 storm, driven by a complex event (CE) resulting of the interaction of two ICMEs. This paper is an extension of a combined study about the solar origins (Balmaceda et al., 2004) and the interplanetary aspects (Echer et al.,2006) of these two sun-earth connection events.

\section{Data Sets}

In order to conduct this analysis, we use geomagnetic indices and ground-based magnetometer data for lowlatitude stations. Geomagnetic indices Dst, ASY-H, SYM$\mathrm{H}$ and $\mathrm{AE}, \mathrm{AU}$ and $\mathrm{AL}$ are obtained from the World Data Center for Geomagnetism. The $\mathrm{Kp}$ index is obtained trough the National Geophysical Data Center (NGDC). A review about the geomagnetic indices can be found in Rostoker (1972), and Iyemori (1990). Time resolution for these indices are 1 hour for Dst, and 1 minute for the ASY-H, SYM-H; AE indices are provisional and have 1 minute resolution.

\section{RESULTS}

Figures 1 and 2 show the magnetic indices during the April 15-18, 1999 and February 10-14, 2000, respectively. The geomagnetic storm development can be followed by the Dst/SYM index in both panels.
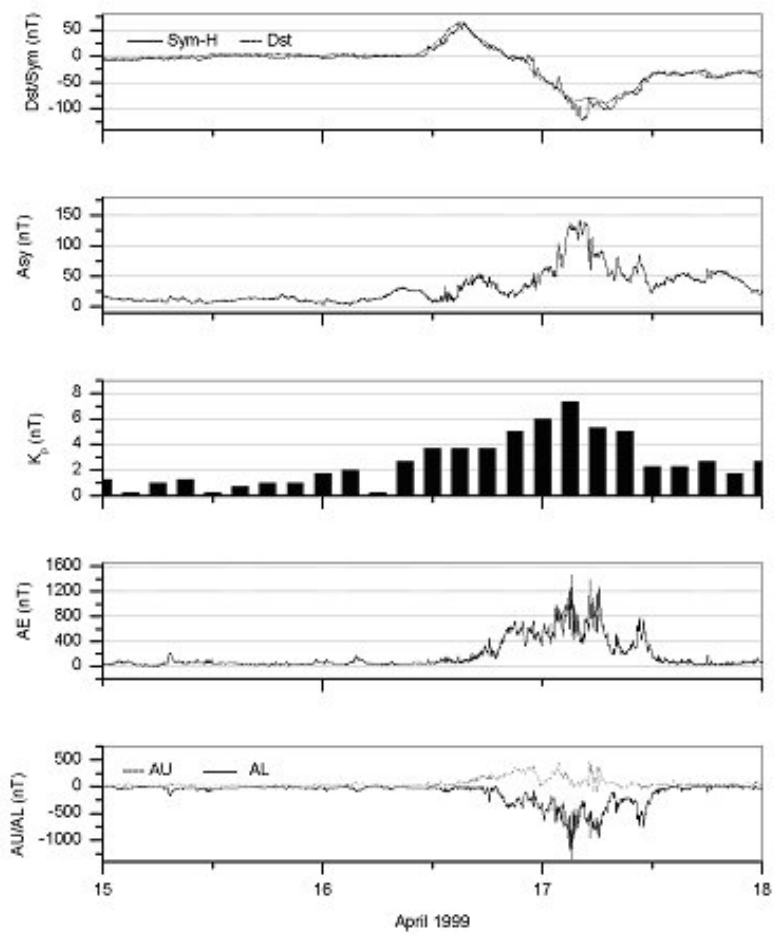

Figure 1: Geomagnetic index data for the April 1999 magnetic storm. Panels are, from top to bottom: Dst and SYM-H, ASY-H, Kp, AE and AU/AL indices. 
The April 1999 magnetic storm was caused by a magnetic cloud. On April 13, 1999, a halo CME earthward directed was observed in LASCO C2 at 03:30 UT with a velocity of $291.2 \mathrm{~km} / \mathrm{s}$ (Balmaceda et al., 2004). The interplanetary remnant of this CME was fast enough to drive an interplanetary shock that reached ACE spacecraft on 10:30 UT April 16, 1999 (Echer et al., 2006). The shock was followed by a S-N magnetic cloud. The very smooth $\mathrm{Bz}$ profile observed in the magnetic cloud have as a consequence the very smooth development of a single step magnetic storm, as observed in Figure 1.
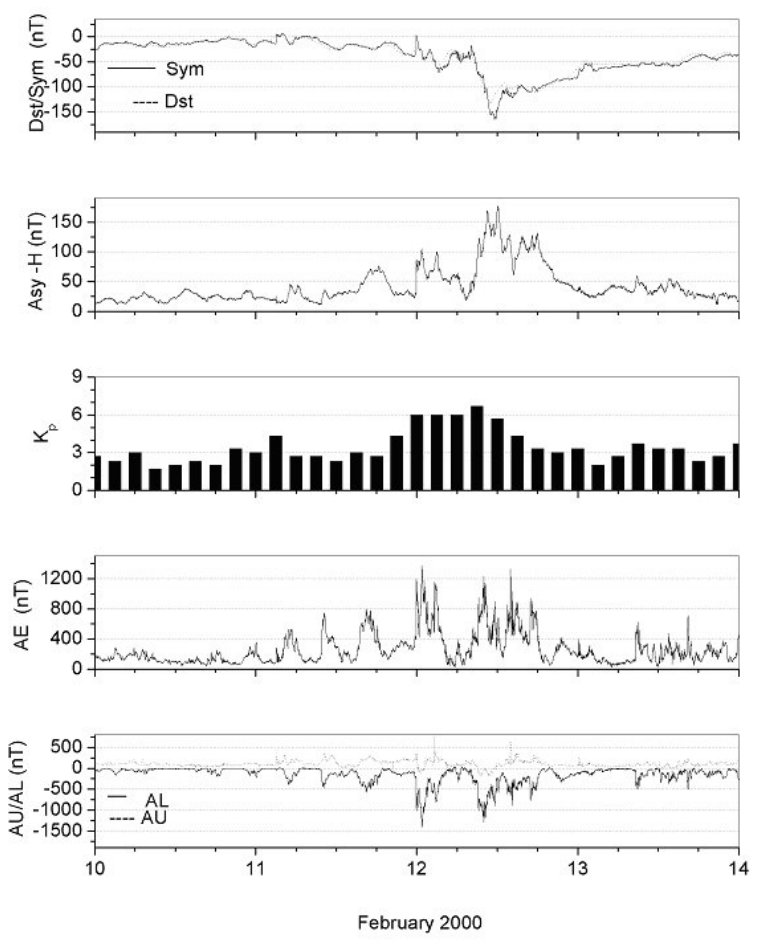

Figure 2: The same as figure 1 for the February 2000 magnetic storm.

The magnetic storm of February 2000 was caused by the interaction of two interplanetary remnants of CMEs. Three CMEs were observed by SOHO on February 8, 9 and 10 2000 (Balmaceda et al, 2004) and the interplanetary remnants of two ICMEs as well as their shocks were observed at ACE on February 2000. The second ICME seems to have engulfed the first one (Echer et al., 2006). The resulting IMF $B_{z}$ profile caused a much more complex magnetic storm, with development in two phases.

Figure 3 presents the solar wind plasma and magnetic field data for the April 16-18, 1999 period. Panels are, from top to bottom: proton temperature $T_{p}$, solar wind speed $V_{s w}$, proton density $N_{p}$, magnetic field azimuth (phi) and inclination (theta) angles, the magnetic field magnitude $B$, and GSM (Geocentric Solar Magnetospheric ) magnetic field components, $B_{x}, B_{y}$, and $B_{z}$, and $\beta$. The continuous vertical line delimits the interplanetary shock ' $S$ ', driven by the MC. The MC boundaries are delimited by the vertical dotted lines, based on the available signatures of the magnetic structure. The region between the shock and the first MC boundary is the sheath, where the plasma is highly turbulent due to shock compression effects.

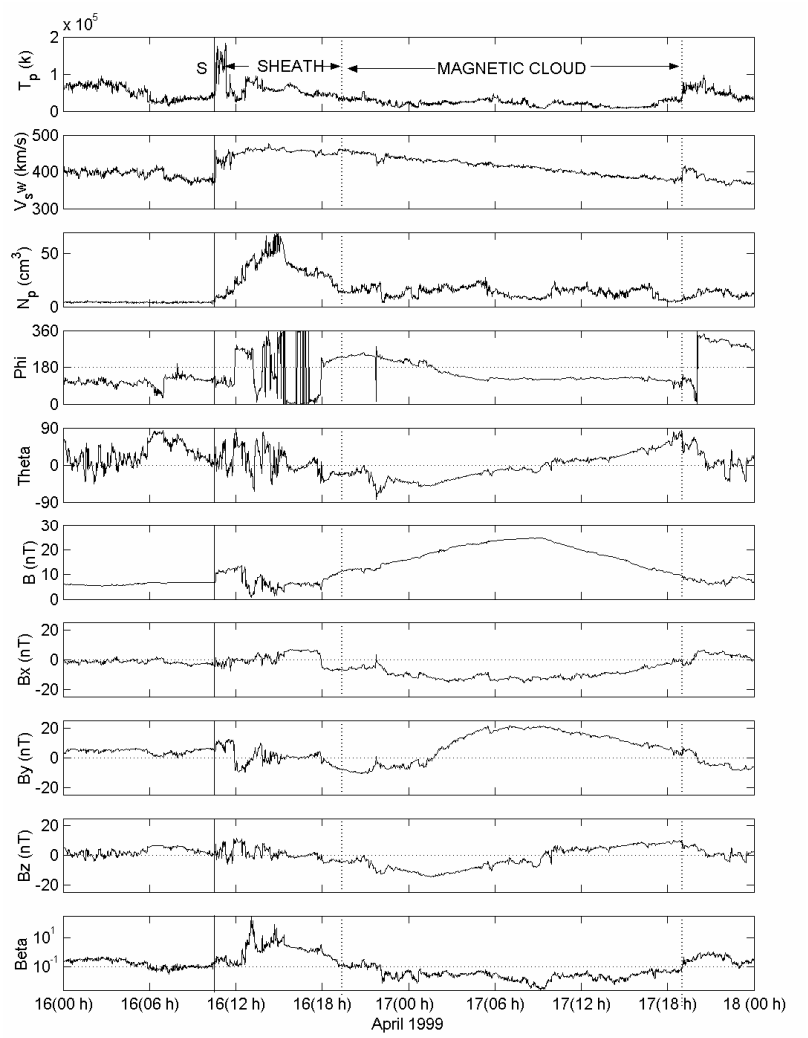

Figure 3. Plasma and interplanetary magnetic field data from ACE spacecraft for the April 16-18, 1999 period.

Figure 3 shows that the $B_{z}$ component have positive or around zero values in the beginning of day 16 . This field configuration leads to a very quiet interval in the $A E$ index (and in both $A U$ and $A L$ ), as shown in Figure 1. Around 18:00 UT on April 16, 1999, the $B_{z}$ component changes to negative values. Around this time, the $A E$ shows an enhancement. The magnetic field maintains the negative $B_{z}$ component until day 17, around 9:00UT. The AE index increases due to this slowly changing structure and exhibits a very high average value, with peaks superposed. From the time of the first increase in AE until around 10:00 UT on day 17, the minimum value registered was $\sim 400 \mathrm{nT}$, with peaks around $1500 \mathrm{nT}$. Even after the end of the interval with negative $B_{z}$ (at 09:00 UT on day 17), there is still some AE activity occurring with peaks around $500 \mathrm{nT}$ at $10 \mathrm{UT}$ and another reaching $\sim 800 \mathrm{nT}$ around 12:00UT. These peaks may be related to substorm occurrences, and since the interplanetary $B_{z}$ field was in the positive domain, they can be part of dissipative processes in the magnetosphere.

For the event on April 1999, before the shock arrival, quiet conditions are observed during days 15 and 16; a sudden impulse/storm sudden commencement is seen in the SYM-H index ( $15 \mathrm{nT})$ and a long duration $(\sim 10 \mathrm{~h})$ initial phase is observed, with positive values of Dst up to +65 nT. This long initial phase was caused by the 
enhanced values of solar wind dynamic pressure and by the absence of a significant southward directed IMF $B_{z}$ component until the beginning of the driven MC. The Dst peak Dst this storm was Dst $=-91 \mathrm{nT}$. The $1 \mathrm{~min}$ resolution SYM-H reached a larger value, of $-123 \mathrm{nT}$. The storm main phase lasted for 10 hours; if we take into account only negative Dst values (16 hours if we include the peak of the initial phase). The Dst index decreases monotonically (in one step) to its peak. The recovery phase to Dst values -30 nT (considered a threshold between quiet and weak magnetic activities, Gonzalez et al., 1994) takes $\sim 15 \mathrm{~h}$. From The ASY-H index we can see that disturbances started with the SSC and reached a peak of $142 \mathrm{nT}$ during the storm main phase. Soon after the storm peak, the ASY-H decreases fast, due to the ring current becomes more symmetric (Liemonhn et al., 2001). The $\mathrm{Kp}$ index shows an increase to moderate values after the SSC, $\mathrm{Kp}+4$ and then increases in accordance with the storm development until a peak of $\sim 70$ during the storm main peak. The Kp decreases from the storm peak is also monotonic. The AE/AU/Al indices show also very low activity before the SSC/storm and an enhanced activity during the storm main phase beginning. Very low activity is seen in the storm initial phase. AE reaches values above $1200 \mathrm{nT}$ during the storm main peak.

Figure 4 presents the plasma and magnetic field interplanetary data for the ICMEs/complex ejecta observed on February 10-14, 2000. Panels present the parameters in the same sequence as in Figure 3. The continuous vertical lines delimit the interplanetary shocks 'S1' and 'S2'. The boundaries of the two ICMEs, 'ICME1' and 'ICME2', are indicated by the vertical dotted lines. The ICME1 is abruptly interrupted by the arrival of the second shock 'S2'. Two CME-related shock fronts passed the ACE spacecraft on February 11.

The interplanetary conditions (mainly those related to the magnetic field) can be contrasted to those during the previous event. While for the first event the $B_{z}$ component of the interplanetary magnetic field shows a smooth and long duration rotation through negative values, in this event the field shows short duration and fast changes, sometimes going from positive to negative values and in the opposite way as well. This pattern leads to a patchy behavior of the AE index, where we observe "packets" of high activity followed by some quiet or almost quiet intervals. This happen mainly along day 11 February 2000 , and beginning of day 12 . The $A E$ peaks reach values around $1400 \mathrm{nT}$, but with low average values. From $\sim 07: 00$ UT to $\sim 17: 00$ UT on day 12 , the $B_{z}$ shows long lasting intervals with negative values. These intervals caused a more continuous increase in the AE index from 0800UT to 1800 UT (although there is a change to positive values during this interval, lasting for a few hours). In the beginning of day 13 , the $A E$ is almost quiet, until some activity starts around 0800 UT, probably related to the Alfvenic-like fluctuations in the interplanetary magnetic field. This patchy behavior in the $A E$ may be related to substorms events caused by the short duration negative $B_{z}$ intervals, which caused short duration reconnection events.

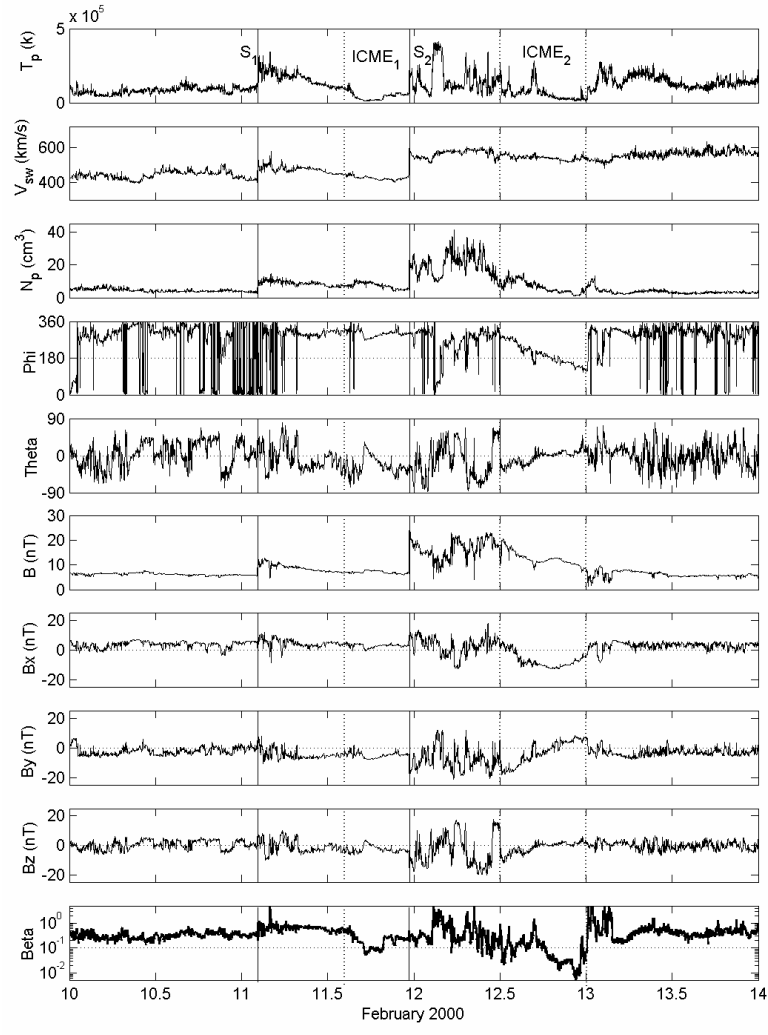

Figure 4. Plasma and interplanetary magnetic field data from ACE spacecraft for the February 10-14 2000 period.

Clearly, the February 2000 storm was more complex as compared to the April 1999. The arrival of the first interplanetary shock at Feb 11, 02:00 UT caused a SI $18 \mathrm{nT}$; the second shock arrival at Feb 11, 23:00 UT caused a larger $\mathrm{SI}, \sim 45 \mathrm{nT}$. During the period between the two SIs, there was a weak magnetic activity (Dst $\sim-25$ $\mathrm{nT}$ ) due to the presence of small and irregular southward interplanetary magnetic field. After the second SI, southward $B_{z}$ were observed (Echer et al., 2006) and Dst reached moderate values of $\sim-60 \mathrm{nT}$, which can be characterized as the first step of this magnetic storm. There was a slight recovery of Dst followed by the main storm peak development during the second ICME period. The total storm main phase, including the 2 steps, takes 11 hours and the peak Dst is $-133 \mathrm{nT}$. The recovery phase is also complex, with an initial recovery being followed by a second peak Dst of $-110 \mathrm{nT}$ after 10 hours. It took $\sim 32$ hours for the Dst reaches quiet values of -30 $\mathrm{nT}$. The behavior of ASY-H index is also complex, with enhancements following the SSCs, the first storm step and then with a peak during the storm peak. The ASY-H decreased initially in the storm recovery phase, increased again during the second peak Dst (pos storm peak) and then decreases fast. The SYM-H peak was -165 nT and the ASY-H peak was $177 \mathrm{nT}$. Kp index shows, in contrast with the April 1999 storm, an enhanced activity during a longer period; after the first $\mathrm{SI} \mathrm{Kp}$ is $\sim 40$ and during the storm main phase is $>60$, reaching a peak $\mathrm{Kp} \sim 7$. Then it decreases monotonically during the storm recovery phase. AE indices are also much more disturbed during the entire interval. Local enhancements are seen during 
the first SI, in the period between the SIs, after the second SI (large increase until peak AE $1200 \mathrm{nT}$ ) and then during the storm main phase with peaks of $\sim 1200$ $\mathrm{nT}$, separated by a decrease.

It is important to have in mind that each index measures a different effect. The $\mathrm{Kp}$ index is considered a global magnetospheric index and it is sensitive to both auroral eletrojet and ring currents; the $A E / A U / A L$ are more sensitive to the ionospheric auroral eletrojets; Dst is sensitive mostly to the ring current, which has a strong asymmetric part during the main phase and a symmetric part more important in the late recovery phase. Further, Dst is also affected by the magnetopause current in the initial phase and by the magnetotail current during the storm. The ASY-H is proportional to the asymmetric ring current part but is also sensitive to auroral currents. Its variation correlates well with the $A E$ index (Iyemori, 1990).

\section{Conclusions}

Based on the magnetic indices, we comparatively analyzed two geomagnetic storms: April, 16-17 1999 (peak Dst $=-91 \mathrm{nT})$ and February 10-12 2000 (peak Dst = $-131 \mathrm{nT}$ ). Two main differences between the two storms can be pointed out, as follows.

The April 1999 is a monotonic, typical one step storm, with a smooth Dst profile. The February 2000 has a more irregular storm profile, with two steps. The storm profiles are basically determined by the driver behavior during both events.

The AE index activity is concentrated around the magnetic storm for the April 1999, but it is very irregular and with several local enhancements during the February 2000 storm. This could mean that the auroral electrojet currents were active since the first shock in the February 2000 storm and remained active even if the ring current was not increasing. The main difference seems to be also caused by the complexity of both storms: April 1999 is a single shock-storm, with south $B_{z}$ just after the SSC and initial phase. The February 2000 had two SIs and a significant negative $B_{z}$ only after the second one. Thus the magnetosphere-ionosphere was disturbed during the period between the two SIs, but not the ring current system, which caused significant AE enhancements. The behavior of the ASY-H and SYM-H indices shows that the asymmetric component is more important around the storm peak. We noticed that for both storms, the ASY-H is enhanced around storm main phase and decreases fast with the recovery phase, similar to the results obtained by Liemoh et al, 2001. The ASY-H is more disturbed for February 2000 as previously mentioned.

\section{Acknowledgments}

The authors would like to thank ACE SWEPAM and MAG teams for make available solar wind plasma and magnetic field data. We thank Kyoto World Data Center for Geomagnetism for Dst and $A E$ indexes and for the ground-based magnetometer data. E. Echer and F. L. Guarnieri thank FAPESP for post-doctoral fellowships (04/11115-4 and 04/14784-4) and financial support research (05/03501-4). E. Echer and M. V. Alves thank also to CNPq for research grants (PQ 2005/3001047 and $P Q$ 303343/2004-4).

\section{References}

Balmaceda, L. A., Gonzalez, W. D., Echer, E., Santos, J. C., Vieira, L.E.A., Dal Lago, A., Guarnieri, F. L. and Gonzalez, A. L. C., The solar origins of the sun-earth connection events on April 1999 and February 2000, Braz. J. Physics, 34, 1745-1747, 2004.

Burlaga, L., Interplanetary Magnetohydrodynamics, 1995.

Clua de Gonzalez, A. L., Mendes da Costa, A. and Gonzalez, W. D., Ring current space-time inhomogeneities in intense geomagnetic storms, Geofisica Internacional, 43, 202-215, 2004.

Dungey, J. W., Interplanetary Magnetic Field and the Auroral Zones, Phys. Rev. Let., 6, 47-49, 1961.

Echer, E, Gonzalez, W. D., Guarnieri F. L., Dal Lago, A. and Vieira, L. E. A., Introduction to Space Weather, Advances in Space Research, 35, 855-865, 2005.

Echer, E., Alves, M. V., Balmaceda, L. A., Guarnieri, F. L., Santos, J. C., Gonzalez, W. D., Dal Lago, A. and Vieira, L. E. A., Interplanetary aspects of the sun-earth connection events on April 1999 and February 2000, Proceedings of the $13^{\text {th }}$ International Congress on Plasma Physics, Kiev, 1-4, 2006.

Gonzalez, W. D., J. A. Joselyn, Y. Kamide, H. W. Kroehl, G. Rostoker, B. T. Tsurutani, and V. M. Vasyliunas, What is a geomagnetic storm?, J. Geophys. Res., 99(A4), 5771-5792, 1994.

Gonzalez, W. D., Tsurutani, B. T. and Clua de Gonzalez, A. L., Interplanetary origin of geomagnetic storms, Space Science Reviews, 88, 529-562, 1999.

lymori, T., Storm-time magnetospheric current inferred from mid-latitude geomagnetic field variations, $\mathrm{J}$. Geomag. Geoeletrc., 42, 1249-1265, 1990.

Liemohn, M. W., Kozyra, J. U., Thomsen, M. F., Roeder. J. L., Lu, G., Borovsky, J. E. and Cayton, T. E., Dominant role of the asymmetric ring current in producing the storm time Dst*, J. Geophys. Res., 106, 10883-10904, 2001.

Rostoker, G., Geomagnetic indexes, Rev. Geophysics, 10, 935, 1972.

Wolf, R. A., Magnetospheric Configuration, in M. G. Kivelson and C. T. Russell, Introduction to Space Physics, Ch. 10, Cambridge University Press, New York, USA, 1995. 